\title{
On the nonexistence of large amplitude stationary solitary waves in symmetric unmagnetized pair plasmas
}

\author{
Frank Verheest ${ }^{1,2}$ \\ ${ }^{1}$ Sterrenkundig Observatorium, Universiteit Gent, Krijgslaan 281, B-9000 Gent, Belgium \\ ${ }^{2}$ School of Physics (Howard College Campus), University of KwaZulu-Natal, Durban 4041, South Africa \\ Received: 14 February 2005 - Revised: 8 April 2005 - Accepted: 11 April 2005 - Published: 9 June 2005 \\ Part of Special Issue "Nonlinear plasma waves-solitons, periodic waves and oscillations"
}

\begin{abstract}
Waves in pair plasmas have a fundamentally different dispersion due to the equal charge-to-mass ratios between negative and positive charges. In view of possible applications e.g. to electron-positron and fullerene pair plasmas, it is shown that there are no stationary large amplitude nonlinear structures in symmetric unmagnetized pair plasmas.
\end{abstract}

\section{Introduction}

Plasmas are an intrinsically nonlinear medium that can support a great variety of waves, far too many to detail here, and discussed in quite a number of classic texts. For ordinary plasmas the great disparities in mass between the negative and positive charge carriers induce quite different time and length scales that can be advantageously exploited to disentangle some of the wave characteristics, not only for linear modes but also for their nonlinear counterparts.

Pair plasmas are radically different from the more usual plasma compositions, because the negative and positive charge carriers have the same mass but opposite charges. Examples of pair plasmas treated in the literature are electronpositron plasmas, of importance in pulsar dynamics and radiation (Sturrock, 1971; Lominadze et al., 1983; Shukla, 1985) and in laboratory studies (Surko et al., 1989; Boehmer et al., 1995; Liang et al., 1998). More recently, pair plasmas were created by using charged fullerenes, consisting of $\mathrm{C}_{60}^{+}$and $\mathrm{C}_{60}^{-}$in equal numbers (Oohara and Hatakeyama, 2003). Fullerenes are molecules containing 60 carbon atoms in a very typical geometric arrangement, and a fullerene pair plasma is a way of mimicking electron-positron plasma behaviour without having to worry about annihilation, so that longer time scales can be considered.

Since the positive and negative charged particles respond on the same scales, the characteristics of waves in pair plas-

Correspondence to: F. Verheest

(frank.verheest@ugent.be) mas cannot always be translated from what obtains in ordinary plasmas by simply letting $m_{i} \rightarrow m_{e}$, where $m_{i}$ and $m_{e}$ are the respective masses of the positive and negative charged particles. According to Oohara and Hatakeyama (2003), the longitudinal-electrostatic modes can be easily measured in pair-ion plasmas, but such linear modes are of the Langmuir type, as is recalled in section 2 below and has also been discussed earlier (Iwamoto, 1993; Zank and Greaves, 1995).

More recently, it has been claimed that ion thermal waves in pair plasmas can be modulated due to their coupling with quasistationary density perturbations (Shukla and Khan, 2005). However, such treatments are weakly nonlinear extensions of linear modes, and it is therefore of interest to investigate what happens to larger amplitude stationary solitary nonlinear waves. As is shown below in Sect. 3, large amplitude stationary solitary wave structures cannot be sustained in "unmagnetized symmetric" pair plasmas, neither of the longitudinal nor of the transverse type. The description of solitons in "magnetized" symmetric pair plasmas has been given in several papers (Berezhiani et al., 1993; Verheest and Cattaert, 2004, 2005), and the present paper indicates that an external magnetic field is a necessary prerequisite for large amplitude stationary solitary structures to exist.

\section{Formalism and invariants}

In unmagnetized plasmas the reference frame can be chosen such that the waves propagate along the $\mathrm{x}$-axis. The model includes the continuity and momentum equations,

$$
\begin{aligned}
& \frac{\partial n_{j}}{\partial t}+\frac{\partial}{\partial x}\left(n_{j} v_{j x}\right)=0, \\
& \frac{\partial \mathbf{v}_{j}}{\partial t}+v_{j x} \frac{\partial \mathbf{v}_{j}}{\partial x}+\frac{1}{n_{j} m} \frac{\partial p_{j}}{\partial x} \mathbf{e}_{x}= \pm \frac{e}{m}\left(\mathbf{E}+\mathbf{v}_{j} \times \mathbf{B}\right),
\end{aligned}
$$

where $n_{j}$ refers to the number densities of the positive ions or positrons $(j=i)$ and of the negative ions or electrons $(j=e)$, both species having charge $e$ in absolute value and mass $m$. Similarly, $p_{j}$ and $\mathbf{v}_{j}$ refer to the respective pressure and fluid 
velocities, while $\mathbf{E}$ and $\mathbf{B}$ are the electric and magnetic fields, respectively. The system is closed by Maxwell's equations,

$$
\begin{aligned}
& \mathbf{e}_{x} \times \frac{\partial \mathbf{E}}{\partial x}+\frac{\partial \mathbf{B}}{\partial t}=\mathbf{0}, \\
& \mathbf{e}_{x} \times \frac{\partial \mathbf{B}}{\partial x}=\frac{1}{c^{2}} \frac{\partial \mathbf{E}}{\partial t}+\mu_{0} e\left(n_{i} \mathbf{v}_{i}-n_{e} \mathbf{v}_{e}\right), \\
& \varepsilon_{0} \frac{\partial E_{x}}{\partial x}=e\left(n_{i}-n_{e}\right),
\end{aligned}
$$

and the parallel magnetic field vanishes, $B_{x}=0$. Parallel and perpendicular are with respect to the direction of wave propagation. The pressures will be assumed polytropic, $p_{i} \propto n_{i}^{\gamma}$ and $p_{e} \propto n_{e}^{\gamma}$, with the same constant of proportionality and the same polytropic index $\gamma$, for reasons of symmetry between the positive and the negative particles.

Before addressing the nonlinear development, we linearize and Fourier transform the relevant Eqs. (1)-(5) for small disturbances varying as $\exp [i(k x-\omega t)]$, with frequency $\omega$ and wave number $k$. We recover two well known results (Zank and Greaves, 1995), the dispersion laws for longitudinal plasma waves,

$\omega^{2}=\omega_{p}^{2}+k^{2} c_{s}^{2}$,

and for transverse electromagnetic modes,

$\omega^{2}=\omega_{p}^{2}+k^{2} c^{2}$.

Remark that the total plasma frequency $\omega_{p}$ in pair plasmas is defined through $\omega_{p}^{2}=2 n_{0} e^{2} / \varepsilon_{0} m$, because both densities simply add up. In addition, $c_{s}$ is the thermal velocity, given through $c_{s}^{2}=\gamma p_{0} / n_{0} m$ in the polytropic description used, and $c$ is the velocity of light.

Next, in view of the nonlinear stationary structures to be studied, we transform $x$ and $t$ to a combined coordinate $\xi=x-V t$, with $V$ the velocity of the stationary solitary structure, and replace all derivatives by the usual chain rule,

$$
\frac{\partial .}{\partial x}=\frac{d .}{d \xi}, \quad \frac{\partial .}{\partial t}=-V \frac{d .}{d \xi},
$$

as used in many studies of nonlinear modes (Adlam and Allen, 1958; Sagdeev, 1966; McKenzie and Doyle, 2003; Sauer et al., 2003; McKenzie et al., 2004; Verheest et al., 2004). In some of these studies $V=0$ has been taken, but then the undisturbed parallel plasma velocities are $-V$, although this shift in point of view does not induce significant changes. Requiring that all variables have this stationarity property rules out envelope solitons where the amplitude profile behaves as a stationary nonlinear hump or dip, but the phase shifts slowly in time. There are, however, nonlinear electromagnetic structures, the oscillitons (Sauer et al., 2003), that superficially look like envelope solitons but have both stationary amplitude and phase. These are, however, outside the scope of the present paper and do not seem to occur in symmetric (magnetized) pair plasmas (Verheest and Cattaert, 2004, 2005).

All derivatives now being with respect to $\xi$, several of the basic equations can be integrated, with the typical boundary conditions at infinity for stationary structures. For e.g. the densities these are

$$
\begin{aligned}
\left.n_{i}\right|_{\xi \rightarrow+\infty} & =n_{0}=\left.n_{e}\right|_{\xi \rightarrow+\infty}, \\
\left.\frac{d n_{i}}{d \xi}\right|_{\xi \rightarrow+\infty} & =0=\left.\frac{d n_{e}}{d \xi}\right|_{\xi \rightarrow+\infty},
\end{aligned}
$$

with analogous conditions for the pressures. The other variables and their derivatives have zero values at infinity. It is to be remarked that periodic structures are thereby excluded, as is typically the case in all Sagdeev-McKenzie treatments.

The continuity Eqs. (1) express conservation of parallel (mass) flux, in its integrated form,

$n_{i}\left(V-v_{i x}\right)=n_{e}\left(V-v_{e x}\right)=n_{0} V$,

and from Faraday's law (3) there follows that

$\mathbf{E}_{\perp}=V \mathbf{B}_{\perp} \times \mathbf{e}_{x}$,

Because the perpendicular components of the equations of motion (2) reduce to

$m \frac{d \mathbf{v}_{j \perp}}{d \xi}= \pm e \mathbf{e}_{x} \times \mathbf{B}_{\perp}$,

the conservation of perpendicular momentum simply is

$\mathbf{v}_{i \perp}+\mathbf{v}_{e \perp}=\mathbf{0}$.

We have shown (Verheest and Cattaert, 2005) that stationary nonlinear structures in symmetric pair plasmas are always charge neutral for propagation parallel to the external magnetic field. A similar proof holds for symmetric unmagnetized pair plasmas, as we briefly sketch for the sake of completeness and readability. We subtract the two parallel equations of motion, contained in Eq. (2),

$$
\begin{aligned}
& m\left(v_{i x}-V\right) \frac{d v_{i x}}{d \xi}+\frac{1}{n_{i}} \frac{d p_{i}}{d \xi}=e\left[E_{x}+\left(\mathbf{v}_{i \perp} \times \mathbf{B}_{\perp}\right) \cdot \mathbf{e}_{x}\right], \\
& m\left(v_{e x}-V\right) \frac{d v_{e x}}{d \xi}+\frac{1}{n_{e}} \frac{d p_{e}}{d \xi}=-e\left[E_{x}+\left(\mathbf{v}_{e \perp} \times \mathbf{B}_{\perp}\right) \cdot \mathbf{e}_{x}\right],
\end{aligned}
$$

from each other, so that only the parallel electric field $E_{x}$ remains on the right hand side, due to Eq. (13). After one derivation with respect to $\xi$ we eliminate $E_{x}$ with the help of Poisson's equation (5) and express all parallel velocities in terms of the densities, using Eq. (10). The result can be written as

$$
\begin{aligned}
& \frac{d^{2}}{d \xi^{2}}\left[\frac{n_{0}^{2} m V^{2}}{2} \frac{1}{n_{i}^{2}}+\frac{\gamma p_{0}}{(\gamma-1) n_{0}^{\gamma}} n_{i}^{\gamma-1}\right]-\frac{2 e^{2}}{\varepsilon_{0}} n_{i} \\
& =\frac{d^{2}}{d \xi^{2}}\left[\frac{n_{0}^{2} m V^{2}}{2} \frac{1}{n_{e}^{2}}+\frac{\gamma p_{0}}{(\gamma-1) n_{0}^{\gamma}} n_{e}^{\gamma-1}\right]-\frac{2 e^{2}}{\varepsilon_{0}} n_{e},
\end{aligned}
$$

given the symmetric polytropic pressures that have in $p_{i} \propto n_{i}^{\gamma}$ and $p_{e} \propto n_{e}^{\gamma}$ the same constant of proportionality and the same polytropic index $\gamma$. Though complicated, Eq. (15) is a second order ordinary differential equation for $n_{i}$, given 
$n_{e}$, or vice versa. It is obvious that Eq. (15) admits $n_{i}=n_{e}=n$ as a solution, and in view of the boundary conditions, this solution is unique. For electron-ion plasmas such a reasoning fails because $m_{i} \neq m_{e}$.

The conservation of mass flux (10) in charge neutral pair plasmas immediately leads to equal parallel velocities, $v_{i x}=v_{e x}=v_{x}$ and from Poisson's equation (5) also to the vanishing of the parallel electric field, $E_{x}=0$, given the conditions at infinity. Since from Eq. (13) we see that $\mathbf{v}_{i \perp}=-\mathbf{v}_{e \perp}=\mathbf{v}_{\perp}$, we can express all fluid quantities in terms of the positive particle variables, omit the species index and get for the parallel equation(s) of motion that

$m\left(v_{x}-V\right) \frac{d v_{x}}{d \xi}+\frac{1}{n} \frac{d p}{d \xi}=e\left(\mathbf{v}_{\perp} \times \mathbf{B}_{\perp}\right) \cdot \mathbf{e}_{x}$.

We now multiply this equation of motion by $n$, use what remains of Ampère's law (4),

$\Gamma \mathbf{e}_{x} \times \frac{d \mathbf{B}_{\perp}}{d \xi}=2 \mu_{0} e n \mathbf{v}_{\perp}$

and flux conservation (10) to derive the parallel momentum invariant

$v_{x}=\frac{\Gamma B_{\perp}^{2}}{4 \mu_{0} n_{0} m V}+\frac{p-p_{0}}{n_{0} m V}$.

Here $\Gamma=1-V^{2} / c^{2}$ is a correcting factor that reduces to 1 in the strictly nonrelativistic limit. However, it plays no essential role in the description and, in view of our using the nonrelativistic equations of motion, it should stay close to 1 anyway.

Finally, we project Eq. (12) upon $\mathbf{v}_{\perp}$ and add this result to Eq. (16). After integration the energy integral is obtained,

$m\left(v_{x}^{2}+v_{\perp}^{2}\right)-2 m V v_{x}+\frac{2 \gamma}{\gamma-1}\left(\frac{p}{n}-\frac{p_{0}}{n_{0}}\right)=0$.

Scalar multiplication of Eq. (12) by $\mathbf{B}_{\perp}$ and vector multiplication of Eq. (17) by $\mathbf{v}_{\perp}$ together give that

$\mathbf{B}_{\perp} \cdot \frac{d \mathbf{v}_{\perp}}{d \xi}=\mathbf{v}_{\perp} \cdot \frac{d \mathbf{B}_{\perp}}{d \xi}=0$,

and consequently

$\mathbf{B}_{\perp} \cdot \mathbf{v}_{\perp}=0$.

Upon using this result in the scalar multiplication of Eq. (17) by $\mathbf{B}_{\perp}$ yields that

$\mathbf{B}_{\perp} \times \frac{d \mathbf{B}_{\perp}}{d \xi}=\mathbf{0}$,

which is only possible when both vectors in this product are parallel, in other words, when the wave magnetic field is linearly polarized. So then is the wave electric field. We thus can orient without loss of generality the reference frame so that $\mathbf{B}_{\perp}=B_{y} \mathbf{e}_{y}$ and $\mathbf{v}_{\perp}=v_{z} \mathbf{e}_{z}$.

\section{Search for nonlinear stationary solitary structures}

To summarize at this stage, we have two remaining first integrals, namely Eqs. (18) and (19), and the nonzero projection of Eq. (17),

$\frac{d B_{y}}{d \xi}=\frac{2 \mu_{0} e}{\Gamma} n v_{z}$.

Since $p$ is polytropic in $n$, and the latter can be expressed in terms of $v_{x}$ with the help of mass conservation (10), there remain three variables to be determined, $v_{x}, v_{z}$ and $B_{y}$, with the help of two algebraic relations and one ordinary differential equation.

To see where this leads us, introduce dimensionless variables as follows: velocities $u_{x, z}=v_{x, z} / V_{0}$, Mach number $M=V / V_{0}$, density $\nu=n / n_{0}$, pressure $\varpi=p / n_{0} m V_{0}^{2}$, length scale $\zeta=e B_{0} \xi / m V_{0}$ and wave magnetic field $b=B_{y} / B_{0}$. We also put $\Gamma=1$ for simplicity, but that could be scaled out if wanted. Here the reference values $V_{0}$ and $p_{0}$ have been connected by $p_{0}=n_{0} m V_{0}^{2}$, so that $V_{0}$ is a measure of the thermal velocity $c_{s}$, up to a factor $\gamma$. Next, $V_{0}$ and $B_{0}$ are connected by $B_{0}^{2}=2 \mu_{0} n_{0} m V_{0}^{2}$ to get rid of all the physical constants. It is seen that this choice for $B_{0}$ corresponds to making $V_{0}$ the Alfvén velocity $V_{A}$, if the plasma were magnetized with a static magnetic strength $B_{0}$.

We express $\varpi$ and $v$ as functions of $u_{x}$ and have to discuss

$$
\begin{aligned}
& u_{x}=\frac{b^{2}}{2 M}+\frac{1}{M}\left[\left(\frac{M}{M-u_{x}}\right)^{\gamma}-1\right], \\
& u_{x}^{2}+u_{z}^{2}-2 M u_{x}+\frac{2 \gamma}{\gamma-1}\left[\left(\frac{M}{M-u_{x}}\right)^{\gamma-1}-1\right]=0, \\
& \frac{d b}{d \zeta}=\frac{M}{M-u_{x}} u_{z} .
\end{aligned}
$$

The two algebraic relations are solved for $u_{z}$ and $b$ as functions of $u_{x}$, values which are then substituted into the ODE in Eq. (24). The resulting expression can be written in the form of an energy integral, to get rid of the square roots and to correlate it with treatments of related nonlinear problems in the Allen (Adlam and Allen, 1958)/Sagdeev (Sagdeev, 1966)/McKenzie (McKenzie and Doyle, 2003) pseudopotential approaches. Hence we obtain

$$
\left(\frac{d u_{x}}{d \zeta}\right)^{2}=F\left(u_{x}\right) \equiv \frac{4 M^{2} G\left(u_{x}\right) H\left(u_{x}\right)}{\left(M-u_{x}\right)^{2}}\left(\frac{d H\left(u_{x}\right)}{d u_{x}}\right)^{-2},
$$

where

$$
\begin{aligned}
& G\left(u_{x}\right)=2 M u_{x}-u_{x}^{2}-\frac{2 \gamma}{\gamma-1}\left[\left(\frac{M}{M-u_{x}}\right)^{\gamma-1}-1\right], \\
& H\left(u_{x}\right)=2 M u_{x}-2\left[\left(\frac{M}{M-u_{x}}\right)^{\gamma}-1\right] .
\end{aligned}
$$

Because $G(0)=H(0)=0$, one sees that $F\left(u_{x}\right)$ and $d F\left(u_{x}\right) / d u_{x}$ both vanish for the initial value $u_{x}=0$. The necessary prerequisites are fulfilled to have a double 


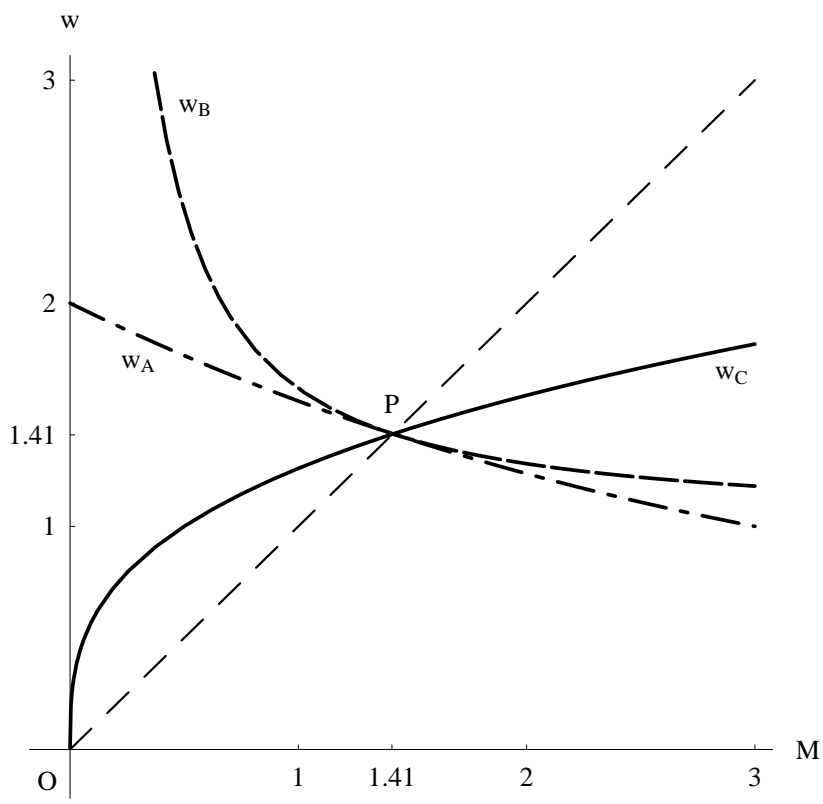

Fig. 1. The graphs for $\gamma=2$ of $w_{A}$ and $w_{B}$ as functions of $M$, representing acceptable roots of the pseudopotential and hence possible soliton amplitudes. The graph of $w_{C}$ represents the asymptote for which the species are infinitely compressed, indicating the limit to possible soliton amplitudes. At a given $M$ and starting on the diagonal (representing the initial value $w=M$ ), the first curve to be encountered determines the existence or not of a stationary solitary wave. It is seen that the asymptote is reached first and no solitons can exist.

root in the origin, and the pseudopotential has the proper convexity there, since

$\frac{d^{2} F}{d u_{x}^{2}}(0)=2 \frac{d G}{d u_{x}}(0) / \frac{d H}{d u_{x}}(0)=8>0$,

at all values of $\gamma \geq 1$ and $M$.

Let us first consider a cold pair plasma, in which all pressure effects are omitted, obtained by formally letting $\gamma \rightarrow 0$, so that only $B_{0}^{2}=2 \mu_{0} n_{0} m V_{0}^{2}$ remains to connect the different dimensional quantities. Then $F\left(u_{x}\right)$ reduces to

$F\left(u_{x}\right)=\frac{2 M u_{x}^{2}\left(2 M-u_{x}\right)}{\left(M-u_{x}\right)^{2}}$.

It is immediately seen that $F\left(u_{x}\right) \rightarrow+\infty$ when $u_{x} \rightarrow M$, whether from below or from above, which from Eq. (10) leads to an infinite compression in both positive and negative species, giving the physical limitation to possible solitary wave amplitudes. As the only other root of $F\left(u_{x}\right)$, outside the double root $u_{x}=0$, is the one in $2 M$, it is inaccessible from the initial conditions and there are no stationary solitary wave structures in symmetric unmagnetized cold pair plasmas. This is in contrast to what occurs in magnetized ones, both at parallel (Verheest and Cattaert, 2004) as well as at oblique or perpendicular (Verheest and Cattaert, 2005) propagation with respect to the direction of the static magnetic field.
We now investigate whether the introduction of finite pressure effects can save the day. Rather than plot the pseudopotential $F\left(u_{x}\right)$ for specific values of $\gamma$ and $M$, as is more commonly done, and discuss its possible roots and poles, we will follow a different approach, explained below. This has the advantage of giving clear answers for all $M$ at specific $\gamma$ values, results which can then be easily visualized for all $\gamma \geq 1$.

Although no special physical significance can be attached to $\gamma=2$, we shall consider this case first, as analytic results are simpler and lead to expressions that are easier to interpret, and hence can guide us for other values of $\gamma$. We write $F\left(u_{x}\right)$ in a mixed notation as

$F\left(u_{x}\right)=2 u_{x}^{2} K(w)$,

explicitly showing the double root in $u_{x}=0$ and where

$K(w)=\frac{w\left(w^{2}+M w-4\right)\left(M w^{2}-w-M\right)}{\left(w^{3}-2 M\right)^{2}}$

has been written in terms of $w=M-u_{x}$. Because initially $u_{x}$ starts from zero, $u_{x} \rightarrow M$ gives an infinite compression in both species, which translates for $w$ into starting from $M$, such that $w>0$ needs always to be observed. In other words, going from $w=M$ to $w=0$ is not an option for physically meaningful solitary structures. The remaining two quadratic factors in the numerator of $K(w)$ in Eq. (30) each have one positive root, namely

$w_{A}=\frac{1}{2}\left[\sqrt{M^{2}+16}-M\right]$,
$w_{B}=\frac{1}{2 M}\left[\sqrt{4 M^{2}+1}+1\right]$,

whereas the denominator of $K(w)$ vanishes for the positive value

$w_{C}=\sqrt[3]{2 M}$.

It can be checked that $w_{A}$ is a zero for $K(w)$ coming from $G\left(u_{x}\right)=0$, hence $u_{z}=0$. Similarly, $w_{B}$ is a zero coming from $H\left(u_{x}\right)=0$, indicating $b=0$, whereas the asymptote in $w_{C}$ corresponds to a zero for $d H\left(u_{x}\right) / d u_{x}$, which is also a zero for $d b / d \zeta$. We will use the same labelling convention also when discussing other values for $\gamma$.

When the curves $w_{A}, w_{B}$ and $w_{C}$ are plotted together as functions of $M$ in Fig. 1, it is seen that they all go through $M=\sqrt{2}$ with the value $\sqrt{2}$, and moreover, the curves for $w_{A}$ and $w_{B}$ are tangent there, in such a way that $w_{A} \leq w_{B}$ for each value for $M$.

The bisectrix represents the line where $w=M$, i.e. the initial values. Hence at a given $M, w$ starts on the bisectrix and goes upward or downward, hoping to reach one of the roots $w_{A}$ or $w_{B}$ of $K(w)$, which would then give the soliton amplitude. Unfortunately, none of these roots can be reached, because the asymptote in $w_{C}$ is hit first, its curve lying closer to the bisectrix than the others. And as indicated already, the curve $w=0$ is physically not acceptable. As in the cold plasma case, there are also here no stationary solitary nonlinear structures in symmetric pair plasmas without an external magnetic field. 


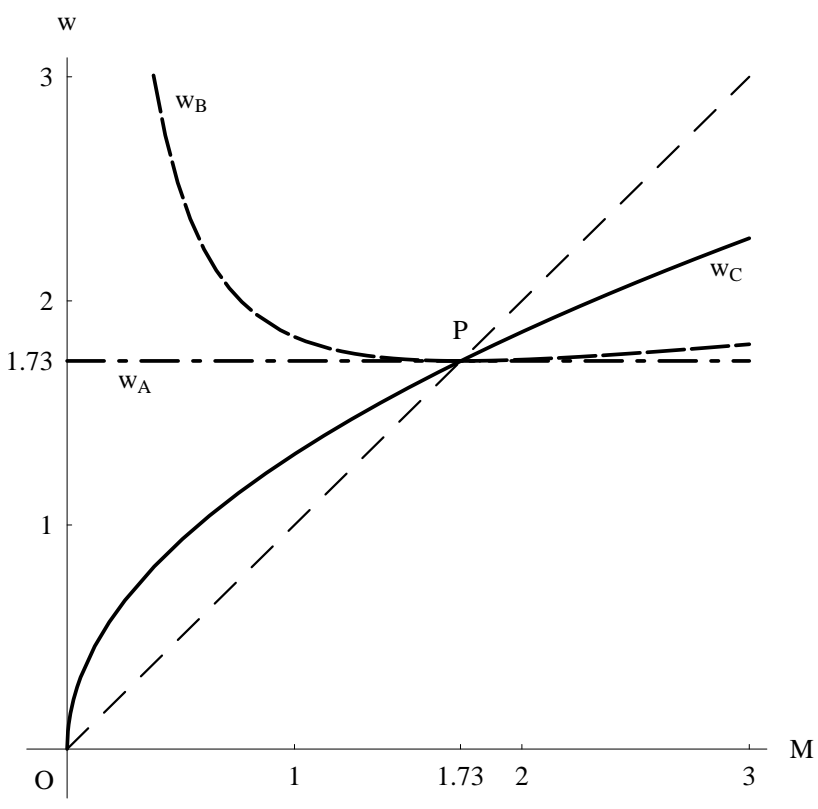

Fig. 2. Analogous curves as in Fig. 1, but for $\gamma=3$, the adiabatic case. It is seen that also here the asymptote is reached first and no solitons can exist.

One can go through the same exercise for the more commonly encountered values $\gamma=1$ (isothermal case) and $\gamma=3$ (adiabatic case), but now some of the curves have to be determined numerically. For $\gamma=3, K(w)$ becomes

$$
K(w)=\frac{w(w+M)\left(w^{2}-3\right)\left(M w^{3}-w^{2}-M w-M^{2}\right)}{\left(w^{4}-3 M^{2}\right)^{2}} .
$$

The admissible roots of $K(w)$ are $w_{A}=\sqrt{3}$ and $w_{B}$ as the only positive root of $M w^{3}-w^{2}-M w-M^{2}=0$, but, as shown in Fig. 2, the pole in $w_{C}=\sqrt[4]{3 M^{2}}$ is always closer to the bisectrix, when $w$ starts from $M$, at any given $M$.

Similarly, $K(w)$ becomes for $\gamma=1$

$K(w)=\frac{w(w M-1)}{\left(w^{2}-1\right)^{2}}\left[M+w-\frac{2 \ln (M / w)}{M-w}\right]$.

The admissible roots of $K(w)$ are $w_{B}=1 / M$ and $w_{A}$ as the only positive root (to be determined numerically) of

$M+w-\frac{2 \ln (M / w)}{M-w}=0$.

The last expression is written in this peculiar form, to indicate that the root $w=M$ (or $u_{x}=0$ ) has to be taken out. Nevertheless, also here the pole in $w_{C}=1$ is always closer to the bisectrix, as indicated in Fig. 3, precluding the existence of stationary solitary waves.

Hence, although the curves are different (some of them becoming a straight line at a constant $w$ value), the conclusion inexorably is the same: there cannot be stationary solitary nonlinear structures in symmetric pair plasmas unless a static magnetic field is present.

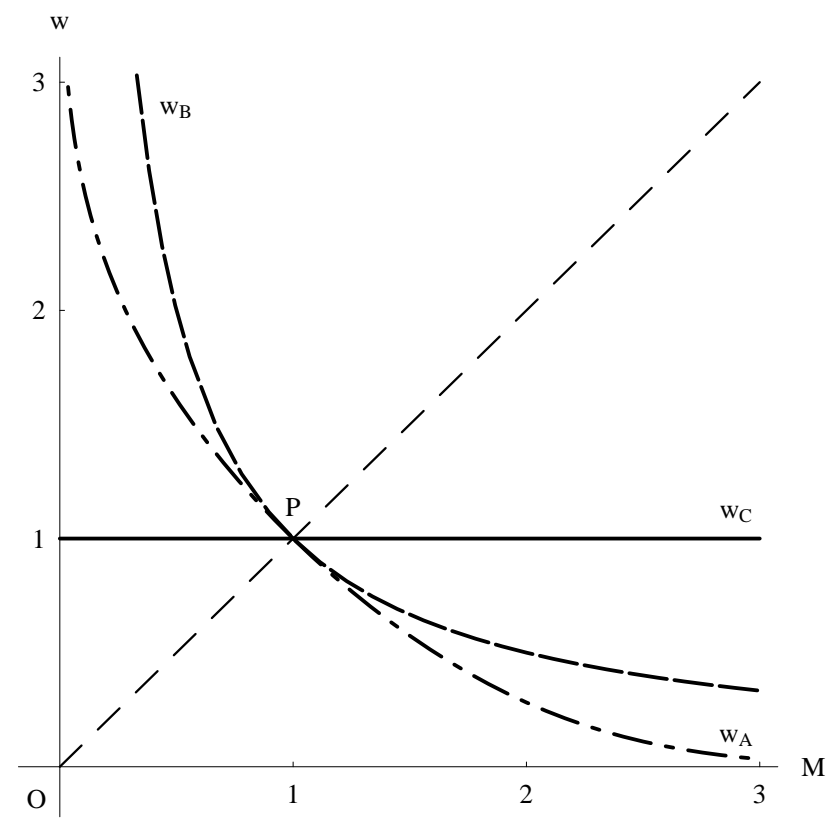

Fig. 3. Analogous curves as in Figs. 1 and 2, but for $\gamma=1$, the isothermal case. Once again, the asymptote is reached first and no solitons can exist.

These conclusions also hold for other values of $\gamma \geq 1$, as briefly indicated now. Rewrite $G\left(u_{x}\right)=0$ as $G(w)=0$ and define $w_{A}$ as the positive root which is different from $w=M$, with an analogous definition of $w_{B}$ coming from $H(w)=0$. One can then show that the curves of $w_{A}$ and $w_{B}$ are tangent in the point $w=M=\sqrt{\gamma}$, with a common slope $(\gamma-3) /(\gamma+1)$. In addition, $w_{A} \leq w_{B}$, although that is not an essential result. However, the curve of $w_{C}$ also goes through $w=M=\sqrt{\gamma}$, but with a quite different slope $(\gamma-1) /(\gamma+1)$. Since

$\frac{\gamma-3}{\gamma+1}<\frac{\gamma-1}{\gamma+1}<1$,

the curve for $w_{C}$ always lies between the diagonal and the curves for $w_{A}$ and $w_{B}$, which corroborates for general $\gamma$ what has been inferred from the three integer values discussed in detail.

\section{Conclusions}

Waves in pair plasmas have a fundamentally different dispersion due to the equal charge-to-mass ratios between negative and positive charges, which mix different timescales. In view of possible applications e.g. to electron-positron and fullerene pair plasmas, we have investigated stationary nonlinear structures in symmetric unmagnetized pair plasmas.

Contrary to what is possible in magnetized pair plasmas under symmetric pressure conditions, large amplitude stationary solitary structures cannot exist in the absence of an external magnetic field. This seems to limit the possible modes in such a system to linear longitudinal, electrostatic 
Langmuir oscillations or transverse, electromagnetic waves (Zank and Greaves, 1995; Oohara and Hatakeyama, 2003), or else to weakly nonlinear modulations of combinations of these (Shukla and Khan, 2005).

Even though immediate experiments are not foreseen for electromagnetic modes in moderately magnetized fullerene pair plasmas, it would be interesting to see whether larger amplitude (electromagnetic) structures could then emerge. Studies of waves in pair plasmas have also been of importance in understanding aspects of pulsars and active galactic nuclei, where the violent surroundings preclude nonlinear phenomena of small amplitude only, and for which the static magnetic field is then an essential ingredient.

Acknowledgements. Interesting discussions with T. Cattaert, M. A. Hellberg, P. K. Kaw, A. Sen and K. Subramanian are gratefully acknowledged. The author has benefitted from the kind hospitality of the International Space Science Institute (Berne, Switzerland), where part of this work germinated during a meeting of the International Team on "Nonlinear Plasma Waves - Solitons, Periodic Waves and Oscillitons in Diverse Space Plasma Environments". The Fonds voor Wetenschappelijk Onderzoek (Vlaanderen) is thanked for a research grant, and the Inter-University Centre for Astronomy and Astrophysics (Pune, India) and the Institute for Plasma Research (Gandhinagar, India) for their kind hospitality during visits where this work was finalized.

Edited by: J. F. McKenzie

Reviewed by: two referees

\section{References}

Adlam, J. H. and Allen, J. E.: The structure of strong collision-free hydromagnetic waves, Philos. Mag., 3, 448-455, 1958.

Berezhiani, V. I., Skarka, V., and Mahajan, S.: Relativistic solitary wave in an electron-positron plasma, Phys. Rev. E, 48, R3252R3255, 1993.

Boehmer, H., Adams, M., and Rynn, N.: Positron trapping in a magnetic mirror configuration, Phys. Plasmas, 2, 4369-4371, 1995.

Iwamoto, N.: Collective modes in nonrelativistic electron-positron plasmas, Phys. Rev. E, 47, 604-611, 1993.
Liang, E. P., Wilks, S. C., and Tabak, M.: Pair production by ultraintense lasers, Phys. Rev. Lett., 81, 4887-4890, 1998.

Lominadze, J. G., Machabeli, G. Z., and Usov, V. V.: Theory of NP 0532 pulsar radiation and the nature of the activity of the Crab nebula, Astrophys. Space Sci., 90, 19-43, 1983.

McKenzie, J. F. and Doyle, T. B.: A unified view of acousticelectrostatic solitons in complex plasmas, New J. Phys., 5, 26.126.10 (2003).

McKenzie, J. F., Dubinin, E., Sauer, K., and Doyle, T. B.: The application of the constants of the motion to nonlinear stationary waves in complex plasmas: a unified fluid dynamic point of view, J. Plasma Phys., 70, 431-462 (2004).

Oohara, W. and Hatakeyama, R.: Pair-ion plasma generation using fullerenes, Phys. Rev. Lett., 91, 205005, 2003.

Sagdeev, R. Z.: Cooperative phenomena and shock waves in collisionless plasmas, in: Reviews of Plasma Physics, edited by: Leontovich, M. A., Consultants Bureau, New York, 4, 23-91, 1966.

Sauer, K., Dubinin, E., and McKenzie, J. F.: Solitons and oscillitons in multi-ion space plasmas, Nonlin. Processes Geophys., 10, 121-130, 2003,

SRef-ID: 1607-7946/npg/2003-10-121.

Shukla, P. K.: Self-modulation of pulsar radiation in strongly magnetized electron-positron plasmas, Astrophys. Space Sci., 114, 381-385, 1985.

Shukla, P. K. and Khan, M.: Envelope ion thermal soliton in a pairion plasma, Phys. Plasmas, 12, 014504, 2005.

Sturrock, P. A.: A model of pulsars, Astrophys. J., 164, 529-556, 1971.

Surko, C. M., Leventhal, M., and Passner, A.: Positron plasma in the laboratory, Phys. Rev. Lett., 62, 901-904, 1989.

Verheest, F. and Cattaert, T.: Large amplitude solitary electromagnetic waves in electron-positron plasmas, Phys. Plasmas, 11, 3078-3082, 2004.

Verheest, F. and Cattaert, T.: Oblique propagation of large amplitude electromagnetic solitons in pair plasmas, Phys. Plasmas, 12, 032304, 2005.

Verheest, F., Cattaert, T., Lakhina, G. S., and Singh, S. V.: Gasdynamic description of electrostatic solitons, J. Plasma Phys., 70, 237-250 (2004).

Zank, G. P. and Greaves, R. G.: Linear and nonlinear modes in nonrelativistic electron-positron plasmas, Phys. Rev. E, 51, 60796090, 1995. 